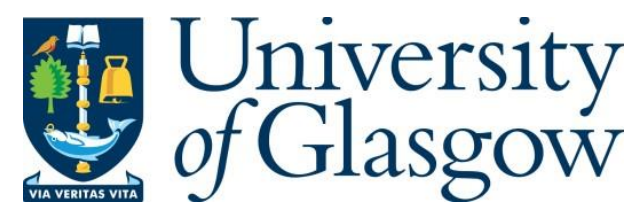

Freeman, E., Griffiths, G. and Brewster, S. (2017) Rhythmic Micro-Gestures: Discreet Interaction On-the-Go. In: 19th ACM International Conference on Multimodal Interaction (ICMI 2017), Glasgow, Scotland, 13-17 Nov 2017, pp. 115119. ISBN 9781450355438.

There may be differences between this version and the published version. You are advised to consult the publisher's version if you wish to cite from it.

(C) The Authors 2017. This is the author's version of the work. It is posted here for your personal use. Not for redistribution. The definitive Version of Record was published in 19th ACM International Conference on Multimodal Interaction (ICMI 2017), Glasgow, Scotland, 13-17 Nov 2017, pp. 115-119. ISBN 9781450355438, http://dx.doi.org/10.1145/3136755.3136815.

http://eprints.gla.ac.uk/147499/

Deposited on: 7 September 2017

Enlighten - Research publications by members of the University of Glasgow http://eprints.gla.ac.uk 


\title{
Rhythmic Micro-Gestures: Discreet Interaction On-the-Go
}

\author{
Euan Freeman, Gareth Griffiths and Stephen Brewster \\ Glasgow Interactive Systems Section \\ University of Glasgow \\ Glasgow, Scotland \\ first.last@glasgow.ac.uk
}

\begin{abstract}
We present rhythmic micro-gestures, micro-movements of the hand that are repeated in time with a rhythm. We present a user study that investigated how well users can perform rhythmic microgestures and if they can use them eyes-free with non-visual feedback. We found that users could successfully use our interaction technique ( $97 \%$ success rate across all gestures) with short interaction times, rating them as low difficulty as well. Simple audio cues that only convey the rhythm outperformed animations showing the hand movements, supporting rhythmic micro-gestures as an eyes-free input technique.
\end{abstract}

\section{CCS CONCEPTS}

\section{•Human-centered computing $\rightarrow$ Gestural input;}

\section{KEYWORDS}

Micro-gestures; rhythmic gestures.

\section{ACM Reference format:}

Euan Freeman, Gareth Griffiths and Stephen Brewster. 2017. Rhythmic Micro-Gestures: Discreet Interaction On-the-Go. In Proceedings of 19th ACM International Conference on Multimodal Interaction, Glasgow, UK, November 13-17, 2017 (ICMI'17), 5 pages.

DOI: $10.1145 / 3136755.3136815$

\section{INTRODUCTION}

Interacting with computing devices is challenging when on-the-go: a user's environment demands lots of attention, traditional forms of input can be difficult whilst walking $[3,12]$, and everyday activities like carrying objects can impair a user's input capabilities [11, 12] Yet, interaction is compelling enough that users are willing to put up with these challenges. Mid-air gestures are one interaction technique that could address many of the problems users experience when interacting on-the-go. The input can be decoupled from the device users are controlling: for example, someone carrying a bag in one hand could gesture with their free hand to control a phone in their pocket, or a runner could gesture without having to reach for their music player. Gestures can also be performed eyes-free, using proprioception, so users can focus on their surroundings.

However, users are not always willing to use gestures, especially in public settings such as when walking or around others, where they are most likely to benefit from them $[1,14]$. This is because gestures might draw unwanted attention, because the hand, arm and body movements may be noticed by others. A more discreet alternative is to use micro-gestures, micro-movements of the hand, which can be performed without attracting attention. Users could perform these small gestures-e.g., tapping the index finger and thumb together-with their hands by the side of their body, allowing them to inconspicuously interact with their devices. Micro-gestures close to the body could also reduce fatigue [8] and have less impact on the user's primary activity [21]

The Midas Touch problem [9] is a well-known problem for gesture interaction, where normal body movements are mistakenly treated as input to a system. This problem arises because the sensing system does not know the difference between normal movements and movements intended to be interactions. This can be especially problematic when users are mobile, e.g., due to natural arm movements while walking or carrying objects. Rhythmic gestures [7] are one solution to the Midas Touch problem: users repeat a gesture in a rhythmic manner, allowing the system to identify the gesture as intentional input. For example, a person carrying shopping might tap their thumb off the side of their hand in time with the rhythm, to get directions to their parked car.

In this paper, we present rhythmic micro-gestures, combining micro-gestures and rhythmic gestures. This is a technique that can be performed discreetly whilst also mitigating the Midas Touch problem. Rhythmic micro-gestures can be used to provide new ways of interacting with devices while on-the-go or in public settings. They could also be used as alternatives to other rhythmic gestures, e.g., to reduce fatigue and increase comfort in VR.

We describe rhythmic micro-gestures and present a study that investigates their usability. We focus on feedback that conveys the rhythm of the gesture and investigate if non-visual feedback is usable, since an eyes-free interaction must be possible without the need for visual cues. We found that users could perform rhythmic gestures quickly and accurately, even when only given audio cues about the rhythm. Our findings support rhythmic micro-gestures as a discreet interaction technique for users on-the-go.

\section{RELATED WORK}

Wolf et al. [20,21] described micro-gestures as small movements of the hands and fingers that can be performed whilst doing another activity. For example, they considered finger movements that could be performed when the hands were grasping a car steering wheel. Chan et al. [5] defined micro-gestures as "detailed gestures in a small interaction space". They focused on the miniaturisation of hand movements to allow users to gesture in more subtle and discreet ways, not necessarily whilst performing another task. Our work is inspired by both definitions of micro-gesture: rhythmic micro-gestures are small hand and finger movements that can be performed subtly whilst also performing other tasks, e.g., when walking, holding shopping bags, or exercising.

Freeman et al. [7] presented rhythmic gestures, purposefully repeated mid-air hand movements that are performed in time with a rhythm: for example, moving a hand from side to side. They investigated their use as a means of initiating interaction with gesture 
systems, since their repetitive nature shows an intent to interact. This overcomes the Midas Touch problem [9], where ordinary movements are misinterpreted as gestures. Addressing this problem is especially important when users are mobile, because of the increase in non-interactive movements (e.g., arms swinging whilst walking). We use the rhythmic gesture concept in this work, applying it instead to micro-gestures.

Rhythmic gestures also allow users to interact from anywhere, because the position of the hand does not matter, only the movement direction and rhythm. This characteristic is also shared by PathSync [4], a gesture technique where users mimic the motion of on-screen cursors using their hands ("motion correlation" [17]). Rhythmic micro-gestures share this property: the movement of the hand and fingers is important, not their position. This means users can perform gestures subtly, e.g., with their hand by their side or behind their back. This may improve willingness to use mid-air gestures, because others are less likely to notice them, misinterpreting them or thinking they are unusual when seen out of context $[1,14]$

Gunslinger [10] is a mid-air gesture technique also motivated by these benefits, where users interact with their hands at the side of their body. They combined hand postures with cursor movement, providing expressive yet inconspicuous input for large displays. Our work is different because we focus on eyes-free gestures that use movement and rhythm to create an expressive input vocabulary.

\section{RHYTHMIC MICRO-GESTURES}

\subsection{Gesture Movements}

Rhythmic micro-gestures are micro-movements of the hand and fingers that are purposefully repeated in time with a rhythm. For example, tapping the index finger and thumb together or rotating the wrist back and forth every $500 \mathrm{~ms}$. The period between a series of movements is the interval, the same terminology used by Freeman et al. [7] for their rhythmic gestures. A rhythmic micro-gesture is successfully performed if the user performs a series of three correct movements in time with the gesture interval. A minimum of three movements offers balance between minimal interaction time and confidence that the user is performing the gesture with intent; this threshold has also been used in related work [7].

We chose four micro-gestures for our study: see Figure 1. These movements were primarily chosen because they could be robustly sensed by our sensing technology in our user study (described later). They can also be performed with the arm by the side of the body, making them inconspicuous and ideal for discreet interaction. They could also be performed whilst on the move: e.g., a runner could gesture to request directions back to their starting point or a person carrying shopping could use a gesture to get a reminder about where their car is parked. These movements only represent a small set of the micro-gesture design space, but the rhythmic gesture concept could be used with all micro-gestures, providing they can be repeated rhythmically.

\subsection{Gesture Feedback}

Visual feedback can be used to show users what movements they can make and at what speed to perform them, to stay in time with the rhythm. Freeman et al. [7] used lights to show the direction of hand movement, with the speed of the animation conveying the 'beat' of the rhythm. Carter et al. [4] used a cursor that traced the outline of a shape to show users how to move their hand. Since we are focused on eyes-free interaction, we wanted to see if users could perform rhythmic micro-gestures in time with just non-visual cues that convey the rhythm.

We used an audio metronome so users could hear the rhythm. The metronome 'ticked' at the start of each beat and users were expected to perform one complete movement in time with the beat. This audio feedback does not convey the specific movement to use. Unlike rhythmic gestures [4, 7], where users are shown multiple available movements at once, we assume that users would know which micro-gesture they wanted to perform; for example, to skip songs in a playlist whilst jogging. Our intention for this style of interaction is that a user would start the rhythmic micro-gesture, then the system would recognise the movement and start the audio metronome, so the user hears the 'beat' they need to follow to complete the gesture. Haptics could be used instead-e.g., a phone in pocket or a smart-watch on the wrist vibrates [6]-but we focus on audio only in this study.

As a control for our study, we created animations that show a virtual hand performing each gesture. These were created by recording data from a Leap Motion sensor, whilst one of the authors performed each gesture. These data were used to control a virtual hand and could be played at different speeds, allowing the same animation to be used for different gesture intervals. A recording of one complete movement was looped for consistency.

\subsection{Apparatus}

We presented the visual feedback on a 15" laptop and the audio feedback was through headphones. The 'tick' sound for the metronome was from a recording of a snare drum.

We used a Leap Motion sensor for tracking users' input during the study. The sensor was placed on a table in front of the users and they performed the gestures over it with their palm facing downwards. Although we intend rhythmic micro-gestures to be performed with the hands by the side of the body or near the torso, this set-up allowed robust and reliable sensing for the experiment. Since the gesture movements only involve wrist or finger articulation, the results should not be affected by arm position. Other sensors, e.g., wearable ones, could be used to recognise these simple gestures. However, our interest in this paper is to test the concept of rhythmic micro-gestures and study their usability, rather than explore tracking options.

\section{USER STUDY}

We ran a user study to investigate if users could perform our rhythmic micro-gestures successfully, to see if they could complete the gestures without visual feedback, and to see how gesture interval affected performance.

There were three within-subjects factors: GUIDE, GESTURE and INTERVAL. There were three types of GUIDE: the audio metronome, the hand animations, and audio and hand animations presented together. There were four GESTUREs, described in the previous section. We chose three INTERVALs: $500 \mathrm{~ms}, 700 \mathrm{~ms}$ and $900 \mathrm{~ms}$. These were used by Freeman et al. in their rhythmic gesture study [7]; we omitted their $1100 \mathrm{~ms}$ interval because our pilot study found it 

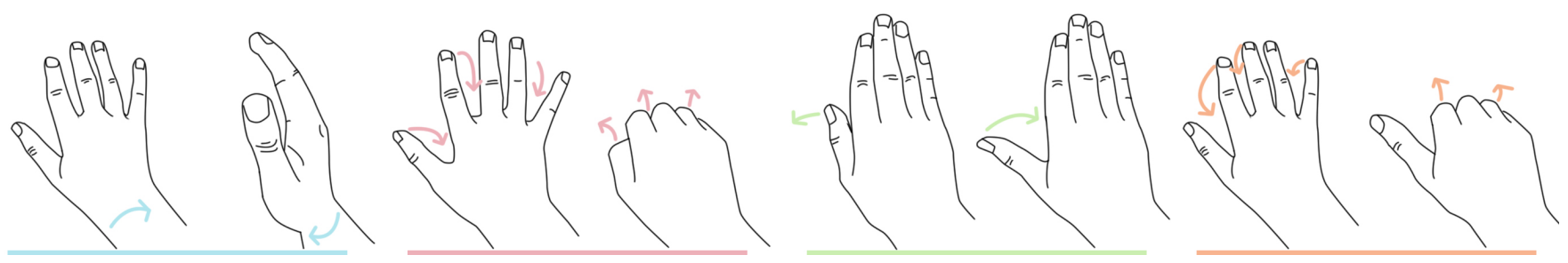

Figure 1: The four micro-gesture movements used in our study. The left and right of each pair show the start and end state of each gesture, respectively. From left to right: (1) Rotate, turning the wrist from side to side; (2) Open, opening and closing the hand to make a fist; (3) Thumb, tapping the side of the hand with the thumb; and (4) Fingers, opening and closing the four fingers so they tap the palm.

\begin{tabular}{llllll}
\hline \multicolumn{2}{c}{ GUIDE } & \multicolumn{2}{c}{ GESTURE } & \multicolumn{2}{c}{ INTERVAL } \\
\hline Metro. & $1945 \mathrm{~ms}$ & Rotate & $2102 \mathrm{~ms}$ & $500 \mathrm{~ms}$ & $2322 \mathrm{~ms}$ \\
Anim. & $2309 \mathrm{~ms}$ & Open & $2135 \mathrm{~ms}$ & $700 \mathrm{~ms}$ & $2127 \mathrm{~ms}$ \\
Both & $2213 \mathrm{~ms}$ & Thumb & $1962 \mathrm{~ms}$ & $900 \mathrm{~ms}$ & $2102 \mathrm{~ms}$ \\
& & Fingers & $2432 \mathrm{~ms}$ & & \\
\hline
\end{tabular}

Table 1: Mean MATch for each factor level, in ms.

was unnecessarily long for the micro-movements we were using. Participants experienced all conditions in a counterbalanced order.

Our experimental task was similar to the task used by Freeman $e t$ al. [7]: participants were asked to perform one of the four GESTURE movements in time with the rhythm. As participants raised their hand over the Leap Motion sensor, the relevant GUIDE was used to convey the INTERVAL (i.e., rhythm) of the gesture. A gesture was successfully matched after three movements in time with the GUIDE (as discussed earlier). Participants had 20 seconds per task.

For each trial, we measured the time taken to successfully match the gesture rhythm (МАТсн) and whether or not the gesture was matched (success). All MATCH times were normalised to $500 \mathrm{~ms}$, allowing fair comparison between INTERVALS. We also asked participants to rate the difficulty of each task (DIFFICULTY), on a scale from 1 (easy) to 10 (difficult); this rating was given verbally.

Fourteen right-handed people participated in this study (four female) with a mean age of 31.3 years ( $\mathrm{sd}=3.7$ years).

\subsection{Results}

4.1.1 Success rates. The mean success rate was $97.2 \%(\mathrm{sd}=3.1 \%)$. Logistic regression was used to analyse the effect of the three factors on sUCCESS. A repeated-measures ANOVA on the regression model found that: GESTURE had a significant effect $\left(\chi^{2}(3)=11.7, \mathrm{p}=0.008\right)$; INTERVAL had a significant effect $\left(\chi^{2}(2)=6.4, \mathrm{p}=0.04\right)$; and GUIDE did not $\left(\chi^{2}(2)=5.9, p=0.05\right)$. No interactions between factors were significant $(\mathrm{p} \geq 0.19)$. Post hoc Wilcoxon's comparisons for GESTURE and INTERVAL found no significant differences (all $\mathrm{p} \geq 0.13$ and $\mathrm{p} \geq 0.1$, respectively).

4.1.2 Time to match gesture. Mean мAтcH time was $2153 \mathrm{~ms}$ ( $\mathrm{sd}=906 \mathrm{~ms})$, normalised to the $500 \mathrm{~ms}$ interval: Table 1 . Times were not normally distributed (Shapiro-Wilk $=0.82, \mathrm{p}<0.001$ ) so the Aligned-Rank Transform [19] was used prior to analysis. The results from a repeated-measures ANOVA are shown in Table 2.

Post hoc t-tests for GUIDE found that metronome led to significantly faster MATCH times than others: both $\mathrm{p} \leq 0.001$. There was no significant difference between others $(\mathrm{p}=0.85)$.

\begin{tabular}{lrrr}
\hline Effect & df & F & p-value \\
\hline GUIDE & 2,441 & 9.6 & $<0.001$ \\
GESTURE & 3,441 & 6.1 & $<0.001$ \\
INTERVAL & 2,441 & 7.0 & 0.001 \\
GUIDE X GESTURE & 6,441 & 1.4 & 0.2 \\
GUIDE X INTERVAL & 4,441 & 0.5 & 0.7 \\
GESTURE X INTERVAL & 6,441 & 2.8 & 0.01 \\
GUIDE X GESTURE X INTERVAL & 12,441 & 2.4 & 0.005 \\
\hline
\end{tabular}

Table 2: ANOVA results for MATсH; $\mathrm{p}<0.05$ highlighted .

\begin{tabular}{lcllll}
\hline \multicolumn{2}{c}{ GUIDE } & \multicolumn{2}{c}{ GESTURE } & \multicolumn{2}{c}{ INTERVAL } \\
\hline Metro. & 2.25 & Rotate & 2.28 & $500 \mathrm{~ms}$ & 2.45 \\
Anim. & 3.04 & Open & 2.60 & $700 \mathrm{~ms}$ & 2.38 \\
Both & 2.31 & Thumb & 2.37 & $900 \mathrm{~ms}$ & 2.78 \\
& & Fingers & 2.89 & & \\
\hline
\end{tabular}

Table 3: Mean difficulty ratings for each factor level.

\begin{tabular}{lrrr}
\hline Effect & df & F & p-value \\
\hline GUIDE & 2,455 & 34.3 & $<0.001$ \\
GESTURE & 3,455 & 12.3 & $<0.001$ \\
INTERVAL & 2,455 & 7.7 & 0.0005 \\
GUIDE X GESTURE & 6,455 & 2.1 & 0.05 \\
GUIDE X INTERVAL & 4,455 & 1.5 & 0.20 \\
GESTURE X INTERVAL & 6,455 & 1.4 & 0.21 \\
GUIDE X GESTURE X INTERVAL & 12,455 & 1.4 & 0.14 \\
\hline
\end{tabular}

Table 4: ANOVA results for DIfFICULTY, $p<0.05$ highlighted .

Post hoc t-tests for GESTURE found that the fingers gesture took significantly more time to match than the thumb $(\mathrm{p}<0.001)$ and rotate $(\mathrm{p}=0.04)$ gestures. No other pairwise comparisons were significant (all $\mathrm{p} \geq 0.1$ ).

Post hoc t-tests for INTERval found that rhythmic micro-gestures with a $900 \mathrm{~ms}$ interval took significantly less time to match than those with a $500 \mathrm{~ms}$ interval $(\mathrm{p}<0.001)$. No other comparisons were significant (both $\mathrm{p} \geq 0.13$ ).

Post hoc t-tests for the interaction between GESTURE and INTERval found no significant differences (all $\mathrm{p} \geq 0.05$ ). Post hoc $\mathrm{t}$-tests for the interaction between all three factors also found no significant differences (all $\mathrm{p} \geq 0.08$ ).

4.1.3 Difficulty ratings. The mean DIFFICULTY rating was 2.5 (sd=1.3): Table 3. Difficulty ratings were transformed using the Aligned-Rank Transform, meaning parametric tests could be used to analyse the non-parametric data [19]. The results from a repeatedmeasures ANOVA are shown in Table 4. 
Post hoc t-tests for GUIDE found that difficulty ratings were higher for the animation condition than for metronome $(\mathrm{p}<0.001)$ and combined conditions $(p<0.001)$. There was no difference between metronome and combined $(\mathrm{p}=0.99)$.

Post hoc t-tests for GESTURE found that difficulty ratings were higher for fingers than thumb $(\mathrm{p}<0.001)$, grasp $(\mathrm{p}=0.006)$, and rotate $(\mathrm{p}<0.001)$. No other comparisons were significant (all $\mathrm{p} \geq 0.06$ ).

Post hoc t-tests for INTERVAL found that difficulty ratings were higher for $900 \mathrm{~ms}$ than for $700 \mathrm{~ms}(\mathrm{p}=0.003)$ and $500 \mathrm{~ms}(\mathrm{p}=0.002)$ intervals; $500 \mathrm{~ms}$ vs $700 \mathrm{~ms}$ was not significant $(\mathrm{p}=0.99)$.

\section{DISCUSSION}

This study investigated if users were able to perform our rhythmic micro-gestures, with visual and non-visual guidance. We intend for these to be discreet, eyes-free gestures for use on-the-go, so it is necessary for users to be able to perform them with non-visual feedback. Related work on similar interactions [4, 7] gave visual cues to convey rhythm and movement, so it was not known if users could perform these with just audio giving the rhythm.

Users gestured successfully, even when only audio guidance was given. Surprisingly, when only audio was used, users needed less time to match the rhythm and also gave lower difficulty ratings. This was unexpected because the visual animation showed how to make the hand movements as well as the movement speeds, which we thought would lead to better performance. Some participants suggested that the visual guide was distracting and it was not clear where the 'beat' occurred. In contrast, audio only conveyed the start of each beat, which may have helped users perceive the rhythm more quickly. Varlet et al. [16] found that discrete audio information was better than continuous visual information when conveying the timing for rhythmic movements, although later work contradicted this [2]; as such, it is not known if our finding can be explained by perception of rhythm in different modalities. However, the important point here is that users can successfully perform our rhythmic micro-gestures without visual attention, supporting their use as an eyes-free interaction technique.

We investigated several rhythmic micro-gestures, comprised of four micro-movements and three gesture intervals. The task success rate was high, with no differences found between the movements and the intervals. Of the four movements, fingers generally took the longest to match and was rated as the most difficult (although was moderately 'easy' at $2.5 / 10$ ). It is unclear why this was more difficult than the others. The only difference between open and fingers is that the thumb does not move in the latter; the gesturesensing algorithm is otherwise the same so we do not think the poor performance was related to sensing issues.

Gestures were performed well with all intervals, with the $900 \mathrm{~ms}$ interval having the best performance (in terms of time needed to match it). Despite this, participants rated it as the most difficult of the three intervals. This may have been because the $900 \mathrm{~ms}$ interval was too slow for the small movements used here. van der Wel et al. [15] found that people tend to avoid making slower hand movements; instead, they prefer to move at their chosen comfortable pace and pause between subsequent movements instead, allowing the slower interval to catch up. This occurs when intervals exceed $800 \mathrm{~ms}$, so $900 \mathrm{~ms}$ may be too long for micro-gestures.
Our rhythmic micro-gestures were successful with $500 \mathrm{~ms}$ intervals. In their study of ordinary rhythmic gestures, Freeman et al. [7] found that simple hand movements were usable with $500 \mathrm{~ms}$ intervals, but more complex movements should use at least $700 \mathrm{~ms}$. We chose a minimum interval of $500 \mathrm{~ms}$ for consistency with their research, but our findings suggest that our eyes-free interactions could also be used with much shorter intervals. Indeed, Repp [13] notes that hand movements can be synchronised with auditory stimulus at much faster speeds than with visual stimulus. More research is required to investigate how well users can perform rhythmic micro-gestures with faster intervals, although the $500 \mathrm{~ms}$ minimum used here is appropriate for most interactions.

Rhythmic micro-gestures are intended for use on-the-go, to enable convenient input in a subtle way. Our lab study was a good first evaluation of usability, demonstrating that we can sense such gestures and that users can perform them well. The next step is to evaluate them 'in the wild', to see how settings like walking affect input performance. Mobility also presents interesting sensing challenges, as body movements will have to be accounted for. We think the ideal location for these gestures is with hands by the side of the body, although the abdomen may also be appropriate (if slightly more conspicuous) for highly mobile users (e.g., runners) [18].

\section{CONCLUSION}

In this paper, we proposed an interaction technique called rhythmic micro-gestures. This technique combines micro-gestures [21], small hand and finger movements, with rhythmic gestures [7], purposefully repeated gestures that avoid the Midas Touch problem and allow the same movements to be used for many actions, by varying the rhythm of movement. Rhythmic micro-gestures can be used for input in a wide variety of situations, but could be particularly effective for interacting while on-the-go. They are inconspicuous and can be performed with hands beside body or out of sight, addressing concerns about gesturing in public $[1,14]$.

We presented a study that investigated how well users could perform rhythmic micro-gestures and if they could perform them with just non-visual feedback, since this would be appropriate for the mobile scenarios we consider. Our findings show that users were able to use our interaction technique successfully, completing $97 \%$ of gestures with short interaction times and rating the interactions as easy. We also found that rhythmic micro-gestures are suitable for the mobile, eyes-free interactions we envision, with the simple audio cues actually outperforming the visual animations.

\section{ACKNOWLEDGEMENTS}

This research was supported by funding from Nokia.

\section{REFERENCES}

[1] David Ahlström, Khalad Hasan, and Pourang Irani. 2014. Are You Comfortable Doing That?: Acceptance Studies of Around-Device Gestures in and for Public Settings. In Proceedings of the 16th International Conference on Human-Computer Interaction with Mobile Devices and Services - Mobile HCI '14. ACM Press, 193-202. DOI : http://dx.doi.org/10.1145/2628363.2628381

[2] Alan Armstrong and Johann Issartel. 2014. Sensorimotor synchronization with audio-visual stimuli: limited multisensory integration. Experimental brain research 232, 11 (2014), 3453-63. DOI : http://dx.doi.org/10.1007/s00221-014-4031-9

[3] Joanna Bergstrom-Lehtovirta, Antti Oulasvirta, and Stephen Brewster. 2011. The effects of walking speed on target acquisition on a touchscreen interface. Proceedings of the 13th International Conference on Human Computer Interaction 
with Mobile Devices - MobileHCI '11 (2011), 143-146. DOI : http://dx.doi.org/10. 1145/2037373.2037396

[4] Marcus Carter, Eduardo Velloso, John Downs, Abigail Sellen, Kenton O'Hara, and Frank Vetere. 2016. PathSync: Multi-User Gestural Interaction with Touchless Rhythmic Path Mimicry. In Proceedings of the SIGCHI Conference on Human Factors in Computing Systems - CHI '16. ACM Press. DOI : http://dx.doi.org/10. $1145 / 2858036.2858284$

[5] Edwin Chan, Teddy Seyed, Wolfgang Stuerzlinger, Xing-Dong Yang, and Frank Maurer. 2016. User Elicitation on Single-hand Microgestures. Proceedings of the 2016 CHI Conference on Human Factors in Computing Systems - CHI '16 (2016), 3403-3414. DOI : http://dx.doi.org/10.1145/2858036.2858589

[6] Euan Freeman, Stephen Brewster, and Vuokko Lantz. 2014. Tactile Feedback for Above-Device Gesture Interfaces: Adding Touch to Touchless Interactions. In Proceedings of the 16th International Conference on Multimodal Interaction - ICMI '14. ACM Press, 419-426. DOI : http://dx.doi.org/10.1145/2663204.2663280

[7] Euan Freeman, Stephen Brewster, and Vuokko Lantz. 2016. Do That, There: An Interaction Technique for Addressing In-Air Gesture Systems. In Proceedings of the 2016 CHI Conference on Human Factors in Computing Systems - CHI '16. ACM Press, 2319-2331. DOI : http://dx.doi.org/10.1145/2858036.2858308

[8] Juan David Hincapié-Ramos, Xiang Guo, Paymahn Moghadasian, and Pourang Irani. 2014. Consumed Endurance: A Metric to Quantify Arm Fatigue of MidAir Interactions. In Proceedings of the SIGCHI Conference on Human Factors in Computing Systems - CHI '14. ACM Press, 1063-1072. http://hci.cs.umanitoba.ca/ projects-and-research/details/ce

[9] Rick Kjeldsen and Jacob Hartman. 2001. Design Issues for Vision-Based Computer Interaction Systems. In Proceedings of the Workshop on Perceptive User Interfaces - PUI '01. ACM Press, 1-8. DOI : http://dx.doi.org/10.1145/971478.971511

[10] Mingyu Liu, Mathieu Nancel, and Daniel Vogel. 2015. Gunslinger: Subtle Arms Down Mid-Air Interaction. In Proceedings of the 28th Annual ACM Symposium on User Interface Software \& Technology - UIST '15. ACM Press, 63-71. DOI http://dx.doi.org/10.1145/2807442.2807489

[11] Alexander Ng, Stephen A. Brewster, and John H. Williamson. 2014. Investigating the effects of encumbrance on one- and two- handed interactions with mobile devices. In Proceedings of the 32nd annual ACM conference on Human factors in computing systems - CHI '14. ACM Press, 1981-1990. DOI : http://dx.doi.org/10. $1145 / 2556288.2557312$

[12] Alexander Ng, John Williamson, and Stephen Brewster. 2015. The Effects of Encumbrance and Mobility on Touch-Based Gesture Interactions for Mobile
Phones. In Proceedings of the 17th International Conference on Human-Computer Interaction with Mobile Devices and Services - MobileHCI '15. ACM Press, 536-546. DOI : http://dx.doi.org/10.1145/2785830.2785853

[13] Bruno H. Repp. 2006. Rate Limits of Sensorimotor Synchronization. Advances in Cognitive Psychology 2, 2 (2006), 163-181. DOI:http://dx.doi.org/10.2478/ v10053-008-0053-9

[14] Julie Rico and Stephen Brewster. 2010. Usable Gestures for Mobile Interfaces. In Proceedings of the SIGCHI Conference on Human Factors in Computing Systems CHI '10. ACM Press, 887-896. DOI : http://dx.doi.org/10.1145/1753326.1753458

[15] Robrecht P. R. D. van der Wel, Dagmar Sternad, and David A. Rosenbaum. 2009. Moving the Arm at Different Rates: Slow Movements are Avoided. Fournal of Motor Behavior 26, 5 (jan 2009), 29-36. DOI : http://dx.doi.org/10.1080/ 00222890903267116

[16] Manuel Varlet, Ludovic Marin, Johann Issartel, R. C. Schmidt, and Benoît G. Bardy. 2012. Continuity of Visual and Auditory Rhythms Influences Sensorimotor Coordination. PLoS ONE 7, 9 (2012), e44082. DOI : http://dx.doi.org/10.1371/ journal.pone. 0044082

[17] Eduardo Velloso, Marcus Carter, Joshua Newn, Augusto Esteves, Christopher Clarke, and Hans Gellersen. 2017. Motion Correlation: Selecting Objects by Matching their Movement. ACM Transactions on Computer-Human Interaction 24, 3 (2017), Article 22. DOI : http://dx.doi.org/10.1145/3064937

[18] Dong-Bach Vo, Eric Lecolinet, and Yves Guiard. 2014. Belly Gestures: Body Centric Gestures on the Abdomen. In Proceedings of the 8th Nordic Conference on Human-Computer Interaction Fun, Fast, Foundational - NordiCHI '14. ACM Press, 687-696. DOI : http://dx.doi.org/10.1145/2639189.2639210

[19] Jacob O. Wobbrock, Leah Findlater, Darren Gergle, and James J. Higgins. 2011. The Aligned Rank Transform for Nonparametric Factorial Analyses Using Only ANOVA Procedures. In Proceedings of the SIGCHI Conference on Human Factors in Computing Systems - CHI '11. ACM Press, 143-146. DOI : http://dx.doi.org/10. 1145/1978942.1978963

[20] Katrin Wolf. 2016. Microgestures - Enabling Gesture Input with Busy Hands. In Peripheral Interaction, Saskia Bakker, Doris Hausen, and Ted Selker (Eds.). Springer, 95-116. DOI : http://dx.doi.org/10.1007/978-3-319-29523-7

[21] Katrin Wolf, Anja Naumann, Michael Rohs, and Jörg Müller. 2011. A Taxonomy of Microinteractions: Defining Microgestures Based on Ergonomic and ScenarioDependent Requirements. In Proceedings of INTERACT '11. Springer Berlin Heidelberg, 559-575. DOI : http://dx.doi.org/10.1007/978-3-642-23774-4_45 